\title{
CARDIOPULMONARY RESUSCITATION
}

RICHARD C. WOLFF, Major, USAF, MC

RICHARD J. WARD, Major, USAF, MC

USAF Hospital Lackland

Aerospace Medical Division (AFSC)

Lackland Air Force Base, Texas

Review 10-62

USAF SCHOOL OF AEROSPACE MEDICINE AEROSPACE MEDICAL DIVISION (AFSC)

BROOKS AIR FORCE BASE, TEXAS

January 1963 